REGARDS

SUR L'ECONOMIE ALLEMANDE

BULLETIN ECONOMIQUE DU CRAC

\section{Regards sur l'économie allemande}

Bulletin économique du CIRAC

$76 \mid 2006$

Varia

\title{
Investissement direct, compétitivité et attractivité
}

\section{Rémi Lallement}

\section{OpenEdition}

\section{Journals}

Édition électronique

URL : http://journals.openedition.org/rea/805

DOI : 10.4000/rea. 805

ISBN : 978-2-8218-0848-5

ISSN : 1965-0787

Éditeur

CIRAC

Édition imprimée

Date de publication : 1 mai 2006

Pagination : 5-12

ISSN : 1156-8992

Référence électronique

Rémi Lallement, "Investissement direct, compétitivité et attractivité », Regards sur l'économie

allemande [En ligne], 76 | mai 2006, document 1, mis en ligne le 16 mai 2008, consulté le 19 avril 2019. URL : http://journals.openedition.org/rea/805 ; DOI : 10.4000/rea.805 


\section{Investissement direct, compétitivité et attractivité}

\section{Rémi Lallement}

Tels qu'ils sont mesurés par les données d'investissement direct (ID), les choix de localisation des entreprises sont souvent considérés comme le révélateur à la fois de la compétitivité des entreprises et de l'attractivité relative des territoires. Or, sur ces deux points, la position actuelle de l'économie allemande semble pour le moins ambiguë, au moins en première analyse. D'un côté, l'arrivée d'A. Merkel au poste de chancelière fédérale, à l'automne 2005, coïncide pour l'économie allemande avec un regain global de compétitivité internationale. En 2005, l'Allemagne a conservé son rang de premier exportateur mondial de marchandises pour la troisième année consécutive, et sa balance commerciale a dégagé un excédent record équivalent à près de $7 \%$ de son PIB. Au plan mondial, qui plus est, elle constitue le seul grand pays industriel dont la part de marché réelle à l'exportation s'est globalement accrue au cours de la dernière décennie. De l'autre côté, les statistiques du chômage entretiennent le pessimisme des Cassandre qui, depuis une dizaine d'années, voient dans l'Allemagne le champion du monde en termes d'exportation... d'emplois. Ils en voient la confirmation dans le fait que, d'année en année, de très nombreuses entreprises allemandes réduisent leurs capacités de production en Allemagne, tout en les étendant à l'étranger.

Dans ce contexte, l'écart qui se manifeste entre l'ampleur des ID allemands à l'étranger et, en sens inverse, celle des ID étrangers en Allemagne - tendanciellement moindre depuis une trentaine d'années - est ainsi souvent interprété comme le symptôme d'une désaffection des entreprises pour le "site Allemagne " (Standort-Deutschland) et, in fine, comme le vecteur d'une perte de substance de l'économie allemande. Ceci nécessite de s'interroger sur la façon dont doivent être appréhendés les liens existant entre l'ID, la compétitivité internationale et l'attractivité des territoires. Or la façon dont les ID entrants et sortants contribuent au développement économique d'un pays varie beaucoup d'un cas à l'autre et dépend pour une large part de la manière dont ces ID sont articulés avec le mode d'organisation socio-économique du pays considéré. Par suite, dans le contexte d'une mondialisation désormais fortement axée sur la mobilité du capital et du savoir, il importe d'examiner la qualité des liaisons productives et cognitives nouées, via l'ID, entre les systèmes nationaux de production et d'innovation en présence (Lallement, 2005).

\section{La position de I'Allemagne à l'égard de I'ID international}

Tout d'abord, la perspective historique conduit à rappeler que, jusqu'au milieu des années 1970, la RFA a davantage abrité d'ID étrangers (ID " entrants ») qu'elle n'a été elle-même à l'origine d'ID à l'étranger (ID " sortants »). Cette situation s'est cependant inversée par la suite, et l'excès en valeur des ID allemands à l'étranger sur les ID étrangers en RFA s'est fortement creusé tout au long des années 1980 et 1990. Sur ce plan, l'Allemagne fait donc désormais partie, avec le Japon, des pays les plus en position d'investisseur net à l'étranger, bien davantage que d'autres pays industriels tels que les Etats-Unis, le Royaume-Uni ou la France.

Investisseur net à l'étranger, comme le Japon 
Faible dynamisme des ID entrants

Au delà des stock ou flux d'ID, indicateurs qui relèvent en grande partie de la sphère financière, surtout pour les sociétés cotées en bourse, l'écart entre les implantations allemandes à l'étranger et les implantations étrangères en Allemagne s'accroît plus nettement encore en termes réels. II est possible d'en juger par l'activité des filiales concernées, par exemple en termes de chiffres d'affaires ou d'effectifs employés. Ainsi et tous secteurs confondus, alors que le nombre d'emplois dans les filiales allemandes à l'étranger équivalait grosso modo à celui des filiales étrangères en RFA en 1980, il l'excédait d'un demi million en 1990, au moment de l'unification, puis de plus d'un million au milieu des années 1990 et de plus de 2,3 millions à la fin 2003 (Bundesbank, 2005).

A ce niveau de généralité, en fait, la singularité de la position allemande vaut moins pour l'évolution des ID sortants que pour celle des ID entrants. Depuis un quart de siècle, en effet, la RFA a en général joué un rôle considérablement moins dynamique comme site d'accueil des multinationales étrangères que comme pourvoyeur d'ID à l'étranger. Dans le total mondial, la part relative de l'Allemagne s'est ainsi située en 2004 à 8,6 \% en termes de stocks d'ID sortants mais de seulement $3,9 \%$ en termes de stocks d'ID entrants. Parmi les 18 pays industriels pour lesquels des données correspondantes sont présentées sur la période 1995-2001 ou des années proches, l'Allemagne constitue même le seul pays industriel dans lequel les filiales industrielles sous contrôle étranger ont enregistré un recul absolu aussi bien en termes de chiffre d'affaires que d'effectifs employés (OECD, 2004).

La part de la RFA comme pays d'origine et d'accueil des ID (en milliards \$ courants)

\begin{tabular}{|c|c|c|c|c|c|c|c|c|c|c|c|c|}
\hline & \multicolumn{6}{|c|}{ Stocks d'ID des pays à l'étranger } & \multicolumn{6}{|c|}{ Stocks d'ID étrangers dans les pays } \\
\hline & 1980 & 1985 & 1990 & 1995 & 2000 & 2004 & 1980 & 1985 & 1990 & 1995 & 2000 & 2004 \\
\hline Allemagne & 43,1 & 59,9 & 151,6 & 258,1 & 541,9 & 833,7 & 36,6 & 36,9 & 111,2 & 192,9 & 271,6 & 348,0 \\
\hline France & 24,3 & 37,8 & 110,1 & 204,4 & 445,1 & 769,4 & 25,9 & 36,7 & 86,8 & 191,4 & 259,8 & 535,2 \\
\hline Royaume-Uni & 80,4 & 100,3 & 229,3 & 304,9 & 897,9 & 1378,1 & 63,0 & 64,0 & 203,9 & 199,8 & 438,6 & 771,7 \\
\hline Etats-Unis & 215,4 & 238,4 & 430,5 & 699,0 & 1316,2 & 2018,2 & 83,0 & 184,6 & 394,9 & 535,6 & 1256,9 & 1473,9 \\
\hline Japon & 19,6 & 44,0 & 201,4 & 238,5 & 278,4 & 370,5 & 3,3 & 4,7 & 9,9 & 36,7 & 50,3 & 97,0 \\
\hline Total partiel & 382,8 & 480,4 & 1122,9 & 1704,9 & 3479,5 & 5369,9 & 211,8 & 326,9 & 806,7 & 1156,4 & 2277,2 & 3225,8 \\
\hline Monde entier & 559,6 & 738,8 & 1785,3 & 2897,6 & 6148,3 & 9732,2 & 692,7 & 972,2 & 1768,6 & 2992,1 & 5780,9 & 8895,3 \\
\hline Part relative RFA & $7,7 \%$ & $8,1 \%$ & $8,5 \%$ & $8,9 \%$ & $8,8 \%$ & $8,6 \%$ & $5,3 \%$ & $3,8 \%$ & $6,3 \%$ & $6,4 \%$ & $4,7 \%$ & $3,9 \%$ \\
\hline
\end{tabular}

Données CNUCED. Sources : pour 1980, 1985, 1995, Lallement (2005) ; pour 1990, 2000 et 2004, UNCTAD (2005) ; calculs de l'auteur. La comparabilité entre ces deux sources n'est que partielle, en raison de changements de définition. Les chiffres pour 2004 sont partiellement des estimations.

Le rôle particulier du Mittelstand

Des considérations d'attractivité mais aussi d'ouverture
Une autre des particularités majeures du cas allemand est qu'en général, les ID allemands à l'étranger sont pour une bonne part le fait d'une multitude d'entreprises indépendantes de taille moyenne - Mittelstand -, qui conservent outreRhin le cœur de leurs activités mais s'implantent de plus en plus fréquemment à l'étranger et notamment dans les PECO proches voire limitrophes de l'Allemagne (Brenken, 2006). Par comparaison, les ID issus de pays tels que les Etats-Unis ou la France sont beaucoup plus, toutes proportions gardées, le fait d'un nombre réduit de grands groupes mondialement connus et dont le centre de gravité se déplace plus nettement vers l'étranger et notamment en direction de pays très distants en termes culturels et géographiques. Ceci étant, il faut souligner que les pays vis-à-vis desquels l'écart de valeur entre ID entrants et sortants est le plus important et s'est le plus creusé pour l'Allemagne, dans la période récente, demeurent dans l'ensemble les pays industriels occidentaux, c'est-à-dire les concurrents traditionnels de l'Allemagne, beaucoup plus que des pays émergents ou à bas salaires.

Ceci renvoie en partie au fait que la plus grande part des ID relève non pas de créations ex nihilo mais d'opérations de fusion-acquisition effectuées entre firmes préexistantes et modifiant davantage la nationalité des entreprises que l'ampleur et la localisation de leurs activités. Or ce rôle dominant des fusionsacquisitions implique que la capacité d'un pays à drainer des ID étrangers sur 
son territoire ne se résume pas à des considérations d'attractivité, c'est-à-dire ne reflète pas uniquement les préférences des investisseurs face aux choix de localisation potentiels qui s'offrent à eux. En effet, elle renvoie aussi à des considérations d'ouverture car elle dépend du degré de facilité avec lequel les entreprises étrangères peuvent effectivement y prendre pied, compte tenu des structures de contrôle du capital observées sur place.

Ceci suggère aussi que, pour l'Allemagne, le creusement de l'écart entre ID entrants et sortants exprime pour une grande part la force compétitive des firmes allemandes et la cohérence du système productif allemand, c'est-à-dire le pouvoir élevé qu'ont les firmes allemandes de prendre le contrôle de firmes étrangères sans être elles-mêmes rachetées par ces dernières. Cette interprétation se retrouve dans l'analyse des régions à l'origine des ID allemands à l'étranger. En effet, la propension à investir à l'étranger se révèle nettement plus élevée de la part des entreprises opérant depuis les Länder opulents et dynamiques du sud de l'Allemagne (Bade-Wurtemberg ou Bavière) que de la part des firmes originaires des régions moins favorisées du nord (par exemple le SchleswigHolstein) ou, a fortiori, en provenance des nouveaux Länder. Ceci est confirmé et précisé par un traitement statistique original effectué à partir de données individuelles provenant du Centre de recherche économique européenne ZEW (Mannheim) et portant sur le secteur manufacturier allemand sur la période 1993-1999. De la part des firmes allemandes concernées, le fait de contrôler des filiales à l'étranger ne témoigne en général pas d'un défaut d'attractivité de leur point d'ancrage et reflète plutôt l'existence de certains avantages compétitifs. Pour elles, il est ainsi significativement lié avec le taux d'exportation, ainsi qu'avec le niveau de productivité du travail et la propension à innover.

En sens inverse, concernant les ID entrants, ces données permettent de montrer qu'en Allemagne, les entreprises faisant partie de groupes 'autochtones' conservent globalement des avantages compétitifs sur les entreprises sous contrôle étranger, du moins dans les secteurs les plus typiques de la spécialisation allemande (construction mécanique, chimie et véhicules de transport), à en juger par un indicateur tel que le taux d'exportation. Des données comparables pour la France font apparaître que les groupes étrangers y font preuve d'une avance compétitive plus générale sur les groupes 'autochtones'.

\section{Les liens entre les ID et le commerce extérieur}

Dans la mesure où la compétitivité de l'économie allemande doit pour une large part être considérée comme la capacité des entreprises allemandes à affirmer leurs positions sur leur marché domestique et sur les marchés mondiaux, il convient de préciser les liens existant entre les ID et le commerce extérieur.

Sur ce plan, de premiers éléments d'analyse concernent les motivations des investisseurs. II en ressort que si, de la part des investisseurs allemands, les ID à l'étranger correspondent de façon croissante à la recherche de moindres coûts de production - et en particulier de main-d'œuvre -, ils continuent néanmoins de renvoyer principalement à des traditionnels motifs d'accès au marché (Buch et al., 2005). En sens inverse, concernant les ID en Allemagne, les firmes étrangères accordent une moins grande importance à des considérations de coût liées à la main-d'œuvre ou à l'énergie (de même qu'à la pression fiscale ou aux aides publiques) qu'à des facteurs de demande et à la qualité de la maind'oeuvre et de certaines infrastructures. En d'autres termes, la dynamique des ID étrangers en Allemagne se révèle en général significativement corrélée avec le rythme relatif de croissance de la demande intérieure allemande mais elle témoigne en partie aussi d'une logique d'accès à des ressources stratégiques, ce qui revient à souligner le poids croissant de facteurs d'offre qui ne se réduisent pas seulement à des considérations de coûts comparatifs. Cette situation ne vaut, du reste, pas que pour l'Allemagne, comme en attestent des
Autre facteur décisif : l'avantage compétitif de l'investisseur

Accéder aux moindres coûts mais aussi aux marchés et à des ressources spécifiques 
Des effets d'éviction à un niveau d'analyse relativement fin

Au plan global, des relations plutôt complémentaires...

... bien qu'à double tranchant

Un fort lien global avec le gonflement de l'excédent commercial allemand travaux récents effectués au MIT (Berger, 2006). Ceci étant, il serait sans doute vain de vouloir opposer les motifs de demande aux motifs d'offre car ils jouent de plus en plus souvent de façon combinée. Par suite, la question des motifs de ces ID ne saurait à elle seule trancher celle de leurs conséquences sur l'évolution du Standort-Deutschland.

Ceci conduit notamment à se demander dans quelle mesure l'essor des ID allemands à l'étranger et des ID étrangers en Allemagne se trouve dans une relation de complémentarité ou, à l'inverse, de substitution, avec celui du commerce extérieur allemand. Or l'analyse met en évidence que ces liens peuvent impliquer des effets d'éviction pour une entreprise donnée, à l'échelle de tel secteur ou vis-à-vis d'un pays précis, c'est-à-dire à un niveau d'analyse relativement fin. A un niveau plus global, par le biais des liaisons intersectorielles amont/aval et au travers d'interdépendances débordant les relations bilatérales vis-à-vis de tel ou tel pays partenaire, ces relations se révèlent cependant complémentaires, le plus souvent.

Concernant les ID allemands à l'étranger, ceci se traduit notamment par le fait qu'il s'agit très souvent d'activités commerciales ou de service implantées par des entreprises industrielles, pour lesquelles de telles implantations sur place constituent un moyen indispensable et puissant d'accéder aux marchés des pays d'accueil. Mis à part ces échanges commerciaux de nature intra-groupe, les entreprises allemandes implantées à l'étranger y font aussi preuve d'une propension assez élevée à s'approvisionner auprès de leurs fournisseurs allemands habituels, a fortiori lorsque ces derniers s'implantent eux mêmes à l'étranger, dans le sillage de leurs clients et donneurs d'ordre. A l'étranger - et notamment dans les PECO -, les unités de production sous contrôle allemand tendent en particulier à faire venir d'Allemagne une grande part des biens d'équipement dont elles ont besoin. Ceci conduit aussi à souligner que, via l'ID à l'étranger, la mondialisation permet aux entreprises allemandes de projeter à l'étranger leurs précieux réseaux de sous-traitance, tout en conservant des attaches profondes avec leur base productive nationale, en partie par le canal plus classique du commerce extérieur. En d'autres termes, la dimension systémique de l'économie allemande se laisse moins que jamais enfermer dans le cadre des frontières nationales: au stade actuel de la mondialisation, les ID contribuent moins à la dissoudre qu'à la modifier géographiquement.

Certes, ce phénomène global de complémentarité se révèle à double tranchant car si, globalement et toutes choses égales par ailleurs, le développement des ID allemands à l'étranger continue d'aller de pair avec un surcroît d'exportations allemandes, il s'accompagne aussi d'une hausse des importations, surtout dans la mesure où les implantations allemandes à l'étranger visent en partie à s'y approvisionner à bon compte. Du côté des ID étrangers en Allemagne, ces phénomènes de complémentarité jouent également, mais plutôt en sens inverse. En effet, les entreprises étrangères utilisent en général leurs implantations outreRhin non seulement comme une porte d'entrée dans le marché intérieur allemand mais aussi comme une base exportatrice - notamment en direction d'autres pays européens -, ce qui confirme que ce pays continue de présenter des avantages de localisation particuliers.

Au total, pour l'Allemagne, les données disponibles suggèrent que le solde des mouvements d'ID entrants et sortants contribue plutôt positivement au solde commercial. En tout cas, et même si corrélation ne vaut pas causalité, le fait que les ID sortants se développent davantage que les ID entrants va structurellement de pair avec une accentuation de l'excédent commercial. Ceci est attesté par une analyse menée sur la période 1995-2002, tant à un niveau sectoriel semi-désagrégé (en 10 secteurs) qu'avec un découpage géographique (en cinq pays ou groupes de pays partenaires). II en ressort tout d'abord clairement qu'en tendance, l'excès grandissant des ID allemands à l'étranger sur les 
ID étrangers en Allemagne va globalement de pair avec un gonflement de l'excédent commercial de ce pays, d'une année sur l'autre.

En moyenne annuelle sur l'ensemble de cette période, il se révèle ensuite que les seuls grands secteurs caractérisés à la fois par un excès des ID sortants sur les ID étrangers entrants et par un excédent commercial sont les plus emblématiques de l'industrie allemande, à savoir l'automobile, la chimie, la construction mécanique et l'électrotechnique (appareils de production et de distribution d'électricité). Symétriquement, les secteurs combinant un excès des ID entrants sur les ID étrangers sortants et un déficit commercial concernent les biens alimentaires, l'électronique (radio-TV, télécommunications, composants), ainsi que les machines de bureau et équipements informatiques : autant de secteurs pour lesquels l'Allemagne est ainsi relativement dominée car doublement pénétrée, à la fois via le commerce extérieur et les ID. De même, l'industrie du textile, de l'habillement et du cuir constitue le seul grand secteur pour lequel l'Allemagne croise un déficit commercial et un (léger) excès des ID sortants sur les ID étrangers entrants ; cette pure logique de délocalisation - au sens de la perte de substance - touche là un domaine qui ne fait pas partie du cœur historique de l'industrie allemande.

De même, et cette fois en termes de pays partenaires, l'Allemagne combine excédent commercial et excès des ID sortants sur les ID étrangers entrants vis-àvis tant des Etats de l'UE que des Etats-Unis, des PVD et des PECO. Le seul groupe de pays faisant exception est constitué principalement des autres pays industriels (dont le Japon) et des pays asiatiques à commerce d'Etat (dont la Chine et le Vietnam); vis-à-vis d'eux, l'Allemagne est globalement engagée dans une véritable logique de délocalisation, au sens où elle associe déficit commercial et position d'investisseur net à l'étranger.

Ces observations en série chronologique et en coupe transversale permettent d'affirmer que l'évolution générale de I'ID tend plutôt à renforcer la spécialisation allemande, ainsi que sa capacité à retirer un surcroît de valeur ajoutée des échanges extérieurs et, en ce sens, à gagner en compétitivité internationale.

\section{Le symptôme d'une dérive vers une « économie de bazar » ?}

Au fil du temps, certes, la valeur totale des exportations allemandes et, plus généralement, de la production réalisée en Allemagne comprend une part croissante de biens importés. Dès lors qu'une part grandissante du made in Germany provient en réalité de l'étranger et en particulier des PECO, la production réalisée sur le sol allemand semble se réduire de plus en plus souvent à un simple montage final. Selon cette thèse, l'Allemagne se trouve de la sorte ravalée désormais au rang « d'économie de bazar » (Sinn, 2004).

Une telle segmentation des processus productifs signifie-t-elle nécessairement une dislocation du système productif allemand? Une interprétation plus positive peut être avancée. Au plan microéconomique, tout d'abord, cet essor de l'entreprise modulaire ouvre de vastes opportunités, par le biais d'une sorte de « Lego industriel »(Berger, 2006). En termes macroéconomiques, en outre, et notamment au plan de la création de richesses et de l'emploi en Allemagne, ce qui compte dans l'ensemble est surtout le fait que l'accroissement du volume des exportations y exerce un effet positif sur le niveau général d'activité. Or, dans le PIB allemand, la part totale de la valeur ajoutée induite par les exportations tend malgré tout à s'accroître, passant d'un peu moins de $20 \%$ en 1991 à environ $22,5 \%$ en 2002, comme l'a calculé le Conseil des Sages (SVR, 2004) sur la base d'un tableau d'entrées/sorties (matrice input/output).

Tout ceci confirme non seulement que les entreprises allemandes réalisant ces exportations sont parvenues à s'insérer avec succès dans la division internationale du travail mais aussi que l'essor des ID participe d'un processus permettant au Standort Deutschland de renforcer ses positions dans la concurrence
Un effet de renforcement mutuel pour la plupart des secteurs...

... et vis-à-vis de la plupart des pays partenaires

Plutôt le signe d'une meilleure insertion dans la division internationale du travail

Une spécialisation accrue sur des critères de compétitivité hors-prix 
mondiale. Dans cette optique, le développement des ID allemands à l'étranger témoigne indéniablement d'une tendance à la délocalisation, surtout en direction de pays à bas salaires d'outre-mer et, désormais, vers les PECO, en particulier pour des segments d'activité intensifs en travail peu qualifié, à faible valeur ajoutée et très sensibles à la concurrence par les coûts. Par ce biais, les entreprises allemandes sont parvenues à améliorer leur compétitivité-prix. Au delà - et ce, tant pour les entreprises allemandes que pour les firmes étrangères installées outre-Rhin -, les ID ont permis au "site Allemagne » de se repositionner en adaptant son appareil productif à la demande domestique et mondiale, en accentuant sa spécialisation sur des critères de compétitivité horsprix : qualité et image de marque des produits, service à la clientèle, respect des délais de livraison, flexibilité et, in fine, capacité d'innovation.

Cela contribue aussi à expliquer le constat que, depuis 1999 et indépendamment de facteurs sectoriels, l'offre d'exportation a bien mieux réagi à l'évolution de la demande mondiale dans le cas de l'Allemagne que dans celui de la France (Gaulier et al., 2005), de sorte que, contrairement à cette dernière, l'Allemagne est parvenue à accroître ses parts du marché mondial sur la période 2000-2004 (Miotti et Sachwald, 2006). Dans cette perspective, l'analyse des liens entre les ID et le commerce international nécessite d'être prolongée par celle des liens entre les ID et les activités d'innovation, en particulier sous l'angle du changement technologique.

\section{Mondialisation technologique et performances du système allemand d'innovation}

Sachant que, par le biais des ID, les entreprises mettent de plus en plus en concurrence les territoires les plus prometteurs au plan de l'innovation, il importe de savoir si et, le cas échéant, en quoi, les mouvements d'ID conduisent pour l'Allemagne à une perte de substance en termes de compétences et de connaissances technologiques ou, à l'inverse, à une concentration, sur place, d'activités à fort contenu en savoir. Dans quelle mesure les décisions d'implantation d'activités d'innovation prises de part et d'autre de la frontière allemande sont-elles dues aux atouts ou aux insuffisances du système allemand d'innovation ? En quoi tendent-elles à les amplifier ou à les réduire ? Dans quelle mesure l'évolution des ID constitue-t-elle un catalyseur du renouvellement technologique en Allemagne?

Un système d'innovation doublement innervé par les ID entrants et sortants
II apparaît tout d'abord que les activités de recherche et développement des filiales étrangères en Allemagne contrebalancent à peu près, par leur ampleur, les activités de $R$ \& D menées par les filiales allemandes à l'étranger. Un raisonnement purement quantitatif et en termes d'équilibre serait toutefois sinon inapproprié, du moins insuffisant. La raison majeure en est que, via I'ID, la mondialisation des activités de $R \& D$ correspond, elle aussi, moins à des phénomènes de substitution (logique de vases communicants) qu'à des effets de complémentarité (jeu à somme positive). Pour une firme donnée, elle permet en effet de créer et contrôler des ressources cognitives utilisables simultanément dans différents pays. Ceci permet de montrer qu'au delà de la seule $R$ \& $D$, le système allemand tire dans l'ensemble parti à la fois des activités d'innovation menées par les filiales allemandes à l'étranger et de celles des filiales étrangères en Allemagne. D'un côté, en effet, les firmes allemandes renforcent généralement leurs avantages compétitifs grâce à des implantations à l'étranger qui leur permettent d'y contrôler certaines ressources technologiques indisponibles outre-Rhin et qui débouchent souvent sur un rapatriement de savoir et parfois aussi, après coup, sur un phénomène plus large de relocalisation en Allemagne (cas des biotechnologies). De l'autre, le territoire allemand profite assez largement de la présence de filiales étrangères intégrées - en général très profondément et souvent d'assez longue date - dans le tissu industriel local et très actives dans le domaine de l'innovation. 
Ces points sont corroborés par un traitement statistique opéré via l'étude comparative de données individuelles provenant essentiellement des volets français et allemands des enquêtes communautaires sur l'innovation (CIS : Community innovation survey). Les résultats suggèrent que le degré d'intégration des groupes étrangers dans le système d'innovation du pays d'accueil est moins élevé dans l'Hexagone qu'outre-Rhin, où le système national d'innovation se révèle plus attractif à bien des égards.

Cette situation d'ensemble ne saurait être attribuée qu'aux seules entreprises ; elle tient aussi aux relations que ces dernières nouent avec certaines institutions caractéristiques du système allemand d'innovation et qui participent elles aussi, à leur façon, à ce processus de mondialisation. A titre d'exemple, les instituts de recherche appliquée de la Fraunhofer Gesellschaft vont parfois jusqu'à s'implanter eux-mêmes à l'étranger, sous la forme non seulement de bureaux de représentation et de bureaux de liaison (notamment dans plusieurs pays d'Asie) mais aussi de véritables centres de recherche (aux Etats-Unis). Au total, qu'elles soient publiques, privées ou mixtes, les institutions allemandes en charge de la science et de la technologie fournissent ainsi aux entreprises multi- ou transnationales des relais rendant possible ou facilitant des échanges bi-directionnels de savoir et de savoir-faire, de part et d'autres de la frontière et, dans l'ensemble, plutôt à l'avantage du système allemand d'innovation. Elles concourent de la sorte à la bonne marche de ce phénomène 'd'intelligence collective' qui caractérise largement le fonctionnement du système allemand.

Le rôle joué par ce cadre institutionnel et ces stratégies d'entreprises explique que, pour ce type de système d'innovation et via l'ID, la mondialisation aille plutôt de pair avec une accentuation de la spécialisation technologique (Lallement, Mouhoud et Paillard, 2002). Cette accentuation peut en partie être décelée sous l'angle sectoriel. Selon certains, cette évolution consacre d'une certaine manière un retour à la "veille économie ». Le fait est que le commerce mondial, depuis l'an 2000 et le dégonflement de la bulle Internet, est tiré principalement par des secteurs relativement traditionnels, tels que les biens d'équipement et la chimie, dans lesquels l'Allemagne est fortement spécialisée (Miotti et Sachwald, 2006). Ceci étant, il y a lieu de se demander si la distinction entre " veille » et " nouvelle économie " a encore un sens, tout comme la distinction faite jadis entre les " secteurs d'avenir » et les "secteurs dépassés ». Il peut être avancé qu'il convient désormais de définir les activités économiques moins par les produits auxquels elles s'appliquent que par leur contenu en savoir et en innovation technologique.

Dès les années 1980, cette situation a été illustrée par le cas de la mécatronique: les entreprises allemandes de la construction mécanique sont parvenues à renforcer leurs positions concurrentielles en intégrant des innovations microélectroniques de provenance étrangère. Depuis quelques années, un phénomène similaire se produit avec le cas déjà mentionné des biotechnologies : les savoirs accumulés dans ce domaine par les grandes firmes allemandes de la chimie-pharmacie, en grande partie via des petites unités de R \& D contrôlées à l'étranger ou sous contrôle étranger en Allemagne, ont permis dernièrement à ce pays de se situer au deuxième rang mondial, derrière les Etats-Unis, en tant que site de production et de développement.

\section{Le rôle d'ensemble des ID dans l'évolution de la compétitivité allemande et du " modèle allemand "}

A l'égard de ces deux types de liens cruciaux - avec le commerce extérieur et avec les activités d'innovation -, les ID s'intègrent ainsi plutôt dans un cercle vertueux pour l'économie allemande, en agissant sur elle comme une sorte de multiplicateur de puissance. Ils lui permettent en effet de davantage structurer son environnement qu'elle n'est elle-même structurée par celui-là. L'ID
Le rôle d'appui de certaines institutions caractéristiques du système allemand

Une contribution au renforcement de la spécialisation allemande

Exemples : mécatronique et biotechnologies 
Vers une transformation du « modèle " plutôt que vers sa disparition international constitue ainsi l'un des moyens privilégiés par lesquels le système allemand de production et d'innovation affirme son ascendant sur d'autres systèmes concurrents à l'échelon régional, en particulier dans les PECO.

Un tel diagnostic conduit à relativiser fortement les nombreux discours prophétisant la disparition progressive du " modèle allemand ", c'est-à-dire annonçant l'irrémédiable dilution du capitalisme allemand au sein d'une économie mondiale à dominante anglo-saxonne. II fait plutôt ressortir la relative plasticité du système allemand de production et d'innovation, sa forte capacité de résilience mais aussi sa transformation progressive. Pour ce "modèle », la question ne se réduit donc pas à l'alternative entre persister et disparaître, qui renvoie à la fiction d'un modèle immuable. Plus intéressante est sans doute la direction prise par les changements en cours qui se traduisent, de proche en proche, par des arrangements institutionnels plus favorables aux entreprises les plus aptes à jouer des différentiels internationaux, du fait de leurs choix de localisation (effectifs ou potentiels). Sous la forme de I'ID, la mobilité croissante des activités de production et d'innovation participe ainsi, pour l'économie allemande, moins d'un changement de trajectoire que d'un changement graduel des modes de production et d'innovation - au sein même du système existant.

TOUT CECI VALIDE AUSSI LA PERTINENCE d'une approche en termes non seulement de "dépendance au sentier » mais aussi d'hybridation entre les modèles, par fertilisation croisée. Par suite, il devient vain de chercher à savoir si, de la sorte, tel modèle national s'aligne sur un autre et, en particulier, si l'Europe se germanise davantage que l'Allemagne ne s'européanise, ou l'inverse, pour paraphraser un propos fameux prononcé par Thomas Mann au lendemain de la Seconde guerre mondiale. En outre, le mode d'organisation socio-économique en vigueur outre-Rhin depuis la naissance de la RFA (1949) se révèle en fin de compte relativement adapté aux nouvelles formes de la mondialisation. Reposant largement sur le principe de subsidiarité, sur une logique de partenariat public/privé, sur l'initiative et l'auto-organisation d'une multitude d'acteurs constitués en réseau, il s'accorde en tout cas mieux que d'autres avec la nature réticulaire de la mondialisation.

\section{Indications bibliographiques :}

BELITZ H., Forschung und Entwicklung in multinationalen Unternehmen, Studien zum deutschen Innovationssystem, $\mathrm{n}^{\circ}$ 8, DIW, janvier 2004

Berger S., Made in Monde : les nouvelles frontières de l'économie mondiale, Paris, 2006

BRENKEN A., Die Globalisierung des Mittelstandes: Chancen und Risiken, Unterlagen zum KfW-Symposium, 13-02-2006, KfW Bankengruppe, Berlin, 2006

Buch C. et al., « Determinants and effects of foreign direct investment : evidence from German firm-level data », Economic policy, n41, janvier 2005

Deutsche Bundesbank, Kapitalverflechtung mit dem Ausland, Statistische Sonderveröffentlichung 10, avril 2005

GaUlier G. LAHRÈChe-RÉVIL A., MÉJeAN I., Dynamique des exportations: une comparaison France-Allemagne, La Lettre du CEPII, ${ }^{\circ}$ 249, octobre 2005

LALLEMENT R., Investissement direct, compétitivité internationale et attractivité des territoires: quelles spécificités pour l'économie allemande?, Université Toulouse I Sciences Sociales, thèse de doctorat de sciences économiques soutenue en décembre 2005

LAllement R., Mouhoud E. M., Paillard S., « Polarisation régionale et internationalisation des activités d'innovation: incidences sur la spécialisation technologique des nations », Région et développement, $\mathrm{n}^{\circ}$ 16, 2002

MIOTTI L., SAChWALD F., Commerce mondial : le retour de la " vieille économie »?, IFRI, février 2006

OECD, Science, Technology and Industry Outlook 2004, Paris, 2004

SINN H.-W., Basar-Ökonomie, ifo-Standpunkt, n 50, 8 janvier 2004

SVR, Erfolge im Ausland - Herausforderungen im Inland, Jahresgutachten 2004/05, (www.sachverstaendigenrat-wirtschaft.de/)

UNCTAD, World Investment Report 2005 - Transnational Corporations and the Internationalization of $R$ \& $D$, New-York/Genève, 2005. 